\title{
Fever of unknown origin in dogs: 50 cases
}

\author{
Mirna Brkljačić́1, Martina Crnogaj ${ }^{1 *}$, Ivana Kišs ${ }^{1}$, Krunoslav Bojanić², Filip Kajin ${ }^{1}$, \\ Iva Šmit ${ }^{1}$, Marin Torti ${ }^{1}$, Ivica Harapin ${ }^{1}$, and Vesna Matijatko ${ }^{1}$ \\ ${ }^{I}$ Clinic for Internal Diseases, Faculty of Veterinary Medicine, University of Zagreb, Zagreb, Croatia \\ ${ }^{2}$ Krunoslav Bojanić, Center of Excellence for Marine Bioprospecting BioProCro, Laboratory for Aquaculture \\ Biotechnology, Ruđer Bošković Institute, Zagreb, Croatia
}

BRKLJAČIĆ, M., M. CRNOGAJ, I. KIŠ, K. BOJANIĆ, F. KAJIN, I. ŠMIT, M. TORTI, I. HARAPIN, V. MATIJATKO: Fever of unknown origin in dogs: 50 cases. Vet. arhiv 90, 255-269, 2020.

\section{ABSTRACT}

Although in veterinary medicine the term "Fever of unknown origin" (FUO) is still liberally used for any febrile patient where no diagnosis has been set after routine diagnostics, the term "true FUO" should be assigned to those cases in which no aetiology is found after extensive diagnostics. The aim of this study was to investigate the occurrence of FUO in dogs, to research the structure of the FUO population, as well as to explore clinical signs and their duration, and the prior treatment and incidence of different diagnoses and their outcomes. Also, this study was conducted in order to explore the amount of diagnostic procedures (with their effectiveness) required to reach a final diagnosis with the intention of helping design and prioritize diagnostics of FUO in dogs in Croatia. Final diagnosis was reached in $94 \%(47 / 50)$ of dogs with a total of 626 diagnostic procedures used. Immune-mediated diseases occurred most frequently $(56 \%)$, followed by infectious diseases $(24 \%)$, neoplastic diseases $(10 \%)$ and congenital diseases $(4 \%)$, with only $6 \%$ of "true FUO". Body temperature ranged between $39.6{ }^{\circ} \mathrm{C}$ and $42.0^{\circ} \mathrm{C}$. The most common symptoms were nonspecific (anorexia and lethargy in $88 \%$, pain in $78 \%$, and gait disorders in $60 \%$ ). The most frequent test performed was hematology while hemoculture was performed the least. Cytology and/or pathohistology were the most effective tests in achieving the final diagnosis (76.32\%). The duration of clinical signs before the diagnosis/ proper treatment was 4-1825 days. The outcome was favorable in 62\%, unfavorable in 32\% and unknown in 6\%.

Key words: fever of unknown origin; fever; pyrexia; dog

\section{Introduction}

Fever of unknown origin (FUO) represents a considerable clinical challenge in both human and veterinary medicine. However, it should be emphasized that the term FUO is certainly overused in veterinary medicine, with too many cases of febrile patients incorrectly and prematurely classified as having FUO, meaning that if they undergo a proper and thorough diagnostic workup, the percentage of patients with no underlying cause of fever should be relatively low.

In human medicine, FUO was defined for the first time in 1961 as a fever over $38.3{ }^{\circ} \mathrm{C}$ that lasts for more than three weeks without certain diagnosis found after 1 week of hospital investigation

\footnotetext{
*Corresponding author:

Assist. Prof. Martina Crnogaj, PhD, DVM, Clinic for Internal Diseases, Faculty of Veterinary Medicine, University of Zagreb, Heinzelova 55, 10000 Zagreb, Croatia, Phone: +385 12390 111, E-mail: martina.crnogaj@vef.hr
} 
(PETERSDORF and BEESON, 1961). Later, this definition was modified in order to include outpatients or inpatients with hospital investigation of a duration of at least 3 days (DURACK and STREET, 1991).

In veterinary medicine, FUO is still not strictly defined, and there are no specific criteria for patient classification in the FUO category. Still, FUO in dogs is usually described as a fever higher than $39.7^{\circ} \mathrm{C}$ to $40.0^{\circ} \mathrm{C}$, with no specific duration (DUNN and DUNN, 1998; BATTERSBY et al., 2006). Generally, in the relevant veterinary small animal internal medicine textbooks, contemporary definitions of FUO vary greatly from the very liberal as a "febrile syndrome for which a diagnosis is not evident" (COUTO, 2014) to the strict and detailed as a "body temperature higher than 39.2 ${ }^{\circ} \mathrm{C}$ with duration of at least three weeks in which no obvious cause is apparent, following at least 3 visits to the veterinarian and/or 3 days of hospitalization, including a basic evaluation of history, clinical examination and minimal diagnostic testing (complete blood count (CBC), serum biochemistry profile and urinalysis)" (RAMSEY i TASKER, 2017). It has been established that fever can be caused by more than 200 diseases in humans (KNOCKAERT et al., 2003). Since a large number of diseases can also cause fever in dogs, the identification of its cause requires a thorough, logical and consistent plan, which should be individually created for each patient, considering the etiology of the fever, clinical signs, the frequency of different pathologies, CBC, biochemistry, urinalysis, radiography and deliberate selection of different diagnostic tests.

When dealing with a patient that has elevated body temperature, the distinction between fever and hyperthermia is of utmost importance. Whilst fever is an elevation in body temperature resulting from a change in the thermoregulatory center, hyperthermia is elevated body temperature resulting from outside causes (i.e. anxiety, environmental conditions, exercise).

Table 1. Differentials in dogs with fever

\begin{tabular}{|c|c|}
\hline Infections & $\begin{array}{l}\text { Bacterial infections } \\
\text { systemic (bacteremia) } \\
\text { local: } \\
\quad \text { abscesses (i.e. prostic, liver, tooth root), pyothorax, pyometra, osteomyelitis, } \\
\text { prostatitis, } \\
\text { discospondylitis, pyothorax, osteomyelitis, cholangitis, septic arthritis, } \\
\text { bacterial endocarditis } \\
\text { other: anaplasmosis, boreliosis, leptospirosis, erlichiosis } \\
\text { Viral infections } \\
\text { canine distemper virus, adenovirus, parvovirus, influenza virus } \\
\text { Fungal infections } \\
\text { aspergillosis, blastomycosis, coccidiomycosis, histoplasmosis } \\
\text { Protozoal infections } \\
\text { babesiosis, leischmaniosis, hepatozoonosis }\end{array}$ \\
\hline Immune-mediated diseases & $\begin{array}{l}\text { Immune-mediated hemolytic anemia, thrombocytopenia, polyarthritits, } \\
\text { polymyositis, steroid-responsive meningitis arteritis, vasculitis, nodular } \\
\text { panniculitis, trapped neutrophil syndrome, polysystemic immune-mediated } \\
\text { diseases (i.e. systemic lupus erythematosus) }\end{array}$ \\
\hline $\begin{array}{l}\text { Inflammatory (non-infectious) } \\
\text { diseases }\end{array}$ & Juvenile cellulitis, myositis, pancreatitis/sterile pancreatic abscesses \\
\hline Neoplastic diseases & $\begin{array}{l}\text { Lymphoproliferative diseases (i.e. leukemia, lymphomas, multiple myeloma), } \\
\text { myeloproliferative diseases, solid tumors, Sertoli cell tumors, lung tumor, } \\
\text { metastatic diseases, any necrotic or infected tumor }\end{array}$ \\
\hline Miscellaneous diseases & $\begin{array}{l}\text { Idiosyncratic reactions to drugs (especially sulfonamides) or vaccines, metaphyseal } \\
\text { osteopathy, panosteitis }\end{array}$ \\
\hline Fever of unknown origin & Unknown etiology after routine or extensive diagnostics \\
\hline
\end{tabular}

Modified from: Causes of fever in dogs and cats. (Ramsey and Tasker, 2017) 
The vast majority of causes of fever can be classified as infectious, immune-mediated or neoplastic (Table 1), among which some are difficult to recognize as well as to diagnose without specific tests due to the nonspecific or even hidden signs (i.e. discospondylitis, steroid responsive meningitis arteritis (SRMA), immune-mediated polyarthritis (IMPA), infectious endocarditis). Some diseases occur more frequently in certain breeds (i.e. SRMA in Beagles and Bernese mountain dogs, immune mediated hemolytic anemia (IMHA) in English cocker spaniels). Furthermore, the patient's travel history should never be neglected (vector borne diseases (VBD) i.e. leishmaniosis). More specifically, in Croatia many VBDs have been confirmed in dogs (babesiosis, borelliosis, anaplasmosis, leishmaniosis, dirofilariosis, hepatozoonosis), some of which are geographically restricted and thus characterized as endemic (i.e. babesiosis in continental parts or leishmaniosis in Dalmatia) (ŽIVIČNJAK et al., 2005; BRKLJAČIĆ et al., 2010; DEŽĐEK et al., 2010; MRLJAK et al., 2017; JURKOVIĆ et al., 2019). Also, data about previous diseases, prior treatments and the response to those treatments should be collected. A thorough physical examination can direct the clinician towards a diagnosis, as well as serving as guidance in choosing further diagnostic tests (i.e. aspiration of enlarged lymph nodes). Physical examination must include orthopedic, neurological and ophthalmological assessment, as well as oral, otic and digital rectal examination.

Previous studies in dogs presenting with pyrexia have demonstrated that the most common cause of pyrexia were immune-mediated disorders (22\% DUNN and DUNN, 1998; 34.8\% BATERSBY et al., 2006; $>50 \%$ BLACK et al., 2018). Infectious diseases are a significantly less frequent cause of FUO in dogs (16\% DUNN and DUNN, 1998; $27.3 \%$ BATTERSBY et al., 2006; 17,9\% BLACK et al., 2018). Contrary to these findings, the occurrence of infections as a cause of FUO in human medicine is much more common: $51 \%$ in children and $35 \%$ in adults (CHOW and ROBINSON, 2011; CHUSID, 2017).

The aim of this study was to investigate the occurrence of FUO in dogs, as well as to describe the structure of the FUO population (age, breed, gender). Furthermore, this study was designed with the purpose of exploring the clinical signs and their duration, prior treatment, the incidence of different diseases causing FUO in dogs, and finally, the outcome. Moreover, we investigated the effectiveness of various diagnostic tests in reaching the final diagnosis, in order to improve the diagnostic approach to FUO in dogs in Croatia.

\section{Materials and methods}

Animals. Among 5768 records of fever in dogs admitted to the Clinic for Internal Medicine, Veterinary Faculty, University of Zagreb, in the period between January 2006 and July 2019, 50 dogs met the following inclusion criteria:

body temperature $\geq 39.6{ }^{\circ} \mathrm{C}$ for at least 3 days complete history data including previous treatments (if treated)

complete clinical examination data

$\mathrm{CBC}$

biochemistry profile

radiography

owner-signed informed consent (for diagnostic and treatment procedures)

unknown diagnosis after initial workup.

Exclusion criteria were:

owner's refusal of recommended diagnostics

any chronic/systemic disease previously diagnosed

Regarding the age at admission, each patient was categorized into one of the following groups: juvenile (2-18 months), young (19-48 months), middle-aged (49-84 months) or older (85-128 months) (Table 4).

Diagnostics. In order to determine the impact of diagnostics in reaching the final diagnosis, each of the following diagnostic procedures: $\mathrm{CBC}$, biochemistry, urinalysis, cytology and/ or pathohistology (PHD), as well as radiography, ultrasound, bacteriology, hemoculture and specific tests (Table 5), was considered as a single test and further classified as: 1) determinant for diagnosis, 2) helpful for diagnosis or 3) meaningless.

Haematology was performed using an $\mathrm{ABC}$ Automated Hematology Analyser (Horiba 
Diagnostics, Montpellier, France) and serum biochemistry using an Olympus AU 640 Analyser (Olympus Diagnostica GmBH, Hamburg, Germany). Thoracic and abdominal radiography was performed using an Eickermeyer EDR HP (IMD Generators s. r. 1., Italy) with digital data processing and image distribution by Agfa CR 30-X (Agfa, Japan). Ultrasound was performed using $\mathrm{s}$ MyLabTM 40Vetusing a 8-6 $\mathrm{mHz}$ microconvex ultrasound transducer (Esaote, Italy).

Synovial and cerebrospinal fluid (CSF) sampling, as well as fine needle aspiration (FNA) of abdominal lymph nodes and parenchymal organs were performed under general anesthesia followed by bacteriology and/or cytology, PHD/ immunocytochemistry. Interpretation of results was done by board certified specialists. Hemoculture and specific tests were performed in external, veterinary accredited laboratories: SYNLAB, Munich, Germany and LABOKLIN, Bad Kissingen, Germany.

Testing for VBD was performed using Idexx SNAP 4Dx Plus Test (Idexx Laboratories, Inc., Westbrook, USA), followed by PCR diagnostic in all samples that tested positive.

All the confirmed diagnoses were divided into five groups, namely: (1) immune-mediated diseases (IMD), (2) infectious diseases (ID), (3) neoplastic diseases (ND), (4) congenital diseases (CD) and (5) FUO ("true FUO") groups (Table 3).

Statistical analysis. All exploratory and statistical data analyses were performed using R software ver. 3.6.0 (R Core Team, 2019). A language and environment for statistical computing. R Foundation for Statistical Computing, Vienna, Austria. URL https://www.R-project.org/). The dependent variable of interest was the disease type (immune-mediated, infectious, neoplastic, congenital, and FUO). The independent variables of interest were demographic (breed, gender, age), anamnestic (clinical symptoms, duration of symptoms, time taken to reach a diagnosis, outcome), and diagnostic test data (hematological and biochemical). Categorical variables were tested for associations using Fisher's exact test for $2 \times 2$ contingency tables, while for lager tables the pairwise nominal independence test was used. Numerical variables (diagnostic test data) were analyzed using linear regression for association with disease type. The association of breeds with FUO diagnosis was tested using the exact binomial test, with the null hypothesis of the probability of a certain breed's proportion in the FUO population being equal to its proportion in the hospital population.

\section{Results}

Cases. Among all records of dogs that presented with fever $(n=5768)$ a total of fifty dogs met the inclusion criteria. Twenty-five breeds were represented in the investigated group, and 24\% were mixed breed (Table 2). The age range was 2-128 months (mean $43 \pm 66.1$ ) (Table 3). There were 18 (36\%) juvenile, 14 (28\%) young, 12 (24\%) middle-aged and 6 (12\%) older dogs (Table 4). In all age groups, IMD was the most frequent diagnosis (Table 4) whereas ID was the second most frequent diagnosis, except in the middle-aged group in which ND was twice as frequent as ID (Table 4). The investigated population consisted of 23 females and 27 males (Table 3). Body temperature ranged between $39.6{ }^{\circ} \mathrm{C}$ and $42.0{ }^{\circ} \mathrm{C}(40 \pm 1.06)$ (Table 3 ). The most common symptoms at admission were nonspecific signs such as general symptoms (loss of appetite/anorexia, lethargy) in $88 \%$ and pain in $78 \%$, followed by gait disorders (lameness, stiffness, painful joints) in $60 \%$ of dogs (Table 3 ).

Diagnostics. Final diagnosis was established in 47 dogs (94\%) with a total of 626 diagnostic procedures used (mean: 12.5 per patient) (Table 5). The most frequent were IMD (56\%) followed by ID in $24 \%$, ND in $10 \%$ and $\mathrm{CD}$ in $4 \%$. The remaining 3 cases $(6 \%)$, in which the underlying disease was not revealed despite extensive diagnostics, were classified as FUO ("true FUO"). Among dogs with IMD $(\mathrm{n}=28)$, the most frequent diagnosis was SRMA (32.1\%), whereas in 2 dogs $(7.1 \%)$ exact diagnosis was not reached despite thorough diagnostics workup, so they were subjected to owner-consented corticosteroid therapy to which they both responded well and thus were categorized as "undefined IMD". In the group with ID, VBD and sepsis were equally represented. 
Table 2. Breed distribution in investigated population

\begin{tabular}{|c|c|c|c|c|c|c|c|}
\hline Breed & $\begin{array}{c}\% \\
\text { of } \mathrm{HP}^{*}\end{array}$ & $\begin{array}{c}\% \\
\text { IP** }\end{array}$ & $\begin{array}{l}\% \\
\text { ID }\end{array}$ & $\begin{array}{l}\% \\
\mathrm{CD}\end{array}$ & $\begin{array}{c}\% \\
\mathrm{tFUO}^{* * *}\end{array}$ & $\begin{array}{c}\% \\
\text { ND }\end{array}$ & $\begin{array}{c}\% \\
\text { IMD }\end{array}$ \\
\hline Alaskan malamute & 0.55 & 2 & 8.33 & 0 & 0 & 0 & 0 \\
\hline American Stafford & 2.47 & 4 & 8.33 & 0 & 0 & 0 & 3.57 \\
\hline Australian shepherd & 0.01 & 2 & 0 & 50 & 0 & 0 & 0 \\
\hline Balkan hound & 0.02 & 2 & 0 & 0 & 0 & 0 & 3.57 \\
\hline Basset & 0.11 & 2 & 8.33 & 0 & 0 & 0 & 0 \\
\hline Beagle & 0.95 & 6 & 0 & 0 & 0 & 0 & 10.71 \\
\hline Bernese mountain dog & 0.82 & 4 & 0 & 0 & 0 & 20 & 3.57 \\
\hline Bischon frise & 0.53 & 2 & 0 & 0 & 0 & 0 & 3.57 \\
\hline Border collie & 0.35 & 2 & 0 & 50 & 0 & 0 & 0 \\
\hline Boxer & 1.66 & 4 & 0 & 0 & 0 & 0 & 7.14 \\
\hline Cane corso & 0.44 & 2 & 0 & 0 & 0 & 0 & 3.57 \\
\hline Chow chow & 0.19 & 2 & 8.33 & 0 & 0 & 0 & 0 \\
\hline English setter & 0.2 & 2 & 8.33 & 0 & 0 & 0 & 0 \\
\hline German shepherd & 4.11 & 10 & 8.33 & 0 & 0 & 0 & 14.29 \\
\hline Golden retriever & 3.54 & 4 & 0 & 0 & 0 & 20 & 3.57 \\
\hline Great Dane & 0.39 & 2 & 0 & 0 & 0 & 0 & 3.57 \\
\hline Greyhound & 0,03 & 2 & 0 & 0 & 0 & 0 & 3.57 \\
\hline Labrador retriever & 5.38 & 6 & 16.67 & 0 & 0 & 0 & 3.57 \\
\hline Maltese & 5.04 & 2 & 0 & 0 & 0 & 0 & 3.57 \\
\hline Miniature schnauzer & 1.06 & 2 & 0 & 0 & 0 & 0 & 3.57 \\
\hline Puli & 0.08 & 2 & 0 & 0 & 0 & 0 & 3.57 \\
\hline Samoyed & 0.77 & 2 & 0 & 0 & 0 & 20 & 0 \\
\hline Siberian husky & 0.65 & 2 & 8.33 & 0 & 0 & & 0 \\
\hline $\begin{array}{l}\text { Tornjak } \\
\text { (Bosnian and Herzegovinian - } \\
\text { Croatian shepherd dog) }\end{array}$ & 0.49 & 4 & 0 & 0 & 0 & 0 & 7.14 \\
\hline Weimaraner & 0.23 & 2 & 0 & 0 & 0 & 0 & 3.57 \\
\hline Mixed breed & 27.22 & 24 & 25 & 0 & 100 & 40 & 14.29 \\
\hline
\end{tabular}

ID-infectious diseases, CD-congenital diseases, ND-neoplastic diseases, IMD-immune-mediated diseases, *HP-overall hospital population of dogs admitted at the Clinic for Internal Diseases, Veterinary Faculty, University of Zagreb, in the period between January 2006 and July 2019 , **IPinvestigated population ( $\mathrm{n}=50$ ), ***tFUO-"true FUO"- fever of unknown origin (dogs with no diagnosis established despite extensive diagnostics). Breeds that are pointed out in bold are breeds that are overrepresented in the investigated population compared to the hospital population ( $\mathrm{P}<0.01)$

All the diagnoses and their frequencies are shown in Table 3. The frequency of procedures used and their effectiveness in reaching the final diagnosis are shown in Table 5. The most frequent test performed was $\mathrm{CBC}$, while the least frequently used was hemoculture. Almost all the tests performed contributed to reaching a diagnosis (either by directing towards, confirming or excluding differentials) except hemoculture, which was performed in just 3 cases and was not helpful in any (because a negative result does not exclude infection/sepsis). Cytology and/or PHD were the most effective tests in achieving the final diagnosis (76.32\%). 


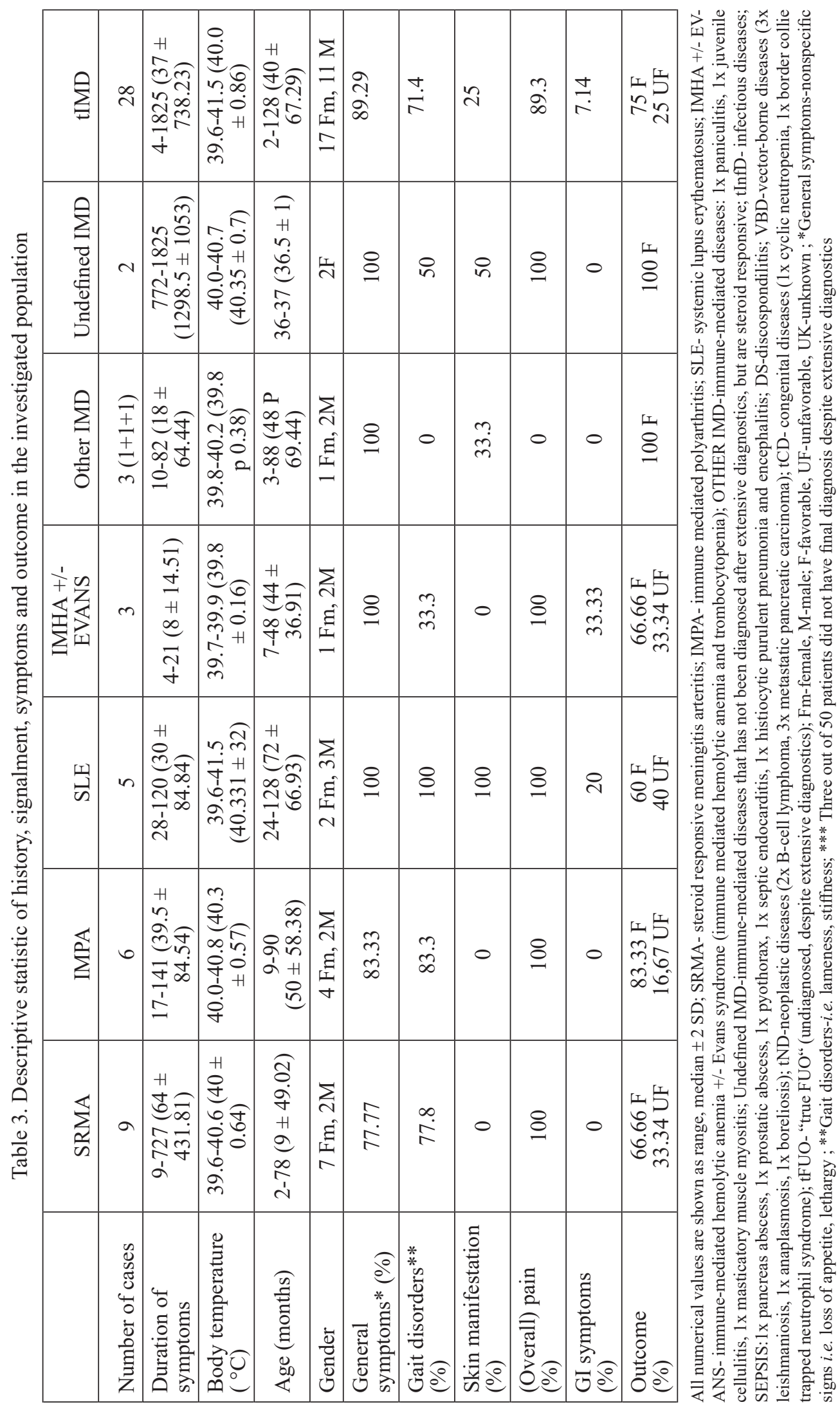




\begin{tabular}{|c|c|c|c|c|c|c|c|c|c|c|c|c|}
\hline 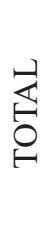 & in & 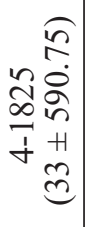 & 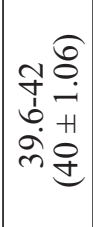 & 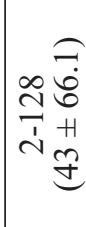 & 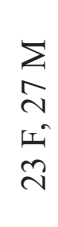 & $\infty$ & 8 & $\stackrel{\infty}{\sim}$ & $\stackrel{\infty}{\sim}$ & $\stackrel{ }{ }$ & 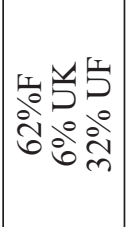 & 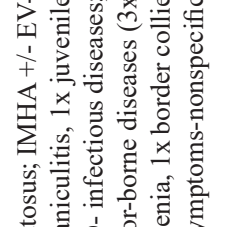 \\
\hline 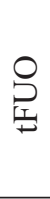 & $n$ & $\stackrel{*}{*}$ & 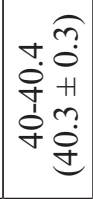 & 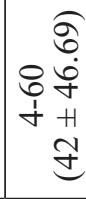 & 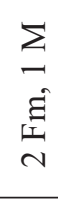 & $\stackrel{8}{ }$ & 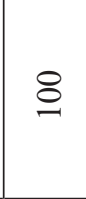 & $\stackrel{m}{m}$ & $\begin{array}{l}0 \\
\ddot{8}\end{array}$ & 0 & $\stackrel{*}{*}$ & 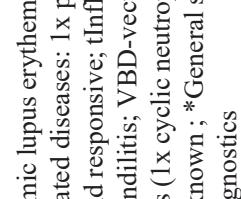 \\
\hline Оి & $N$ & 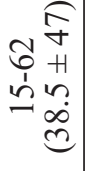 & 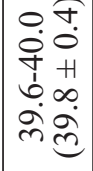 & 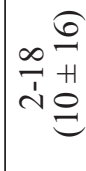 & $\sum_{N}$ & in & in & in & 0 & 0 & 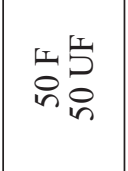 & 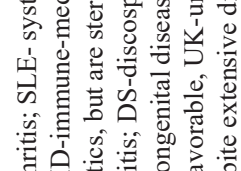 \\
\hline 要 & in & 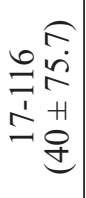 & 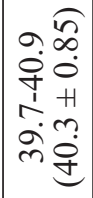 & 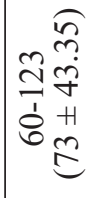 & $\begin{array}{l}\sum_{m} \\
\xi \\
\text { İ } \\
\text { N }\end{array}$ & $\infty$ & 0 & 0 & 8 & $\stackrel{P}{+}$ & $\begin{array}{l}\omega_{1}^{L} \\
\text { ¿ }\end{array}$ & 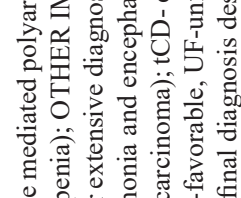 \\
\hline 突 & $\simeq$ & 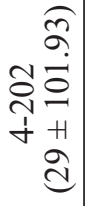 & 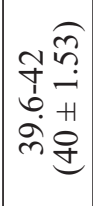 & 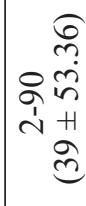 & 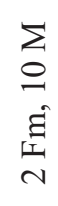 & 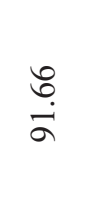 & 으 & $\stackrel{\stackrel{8}{\circ}}{\vec{\gamma}}$ & $\stackrel{2}{2}$ & $\stackrel{m}{\infty}$ & 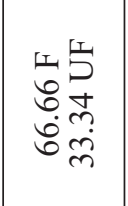 & 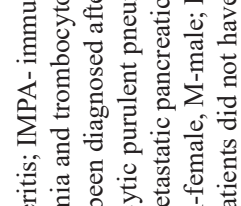 \\
\hline$\stackrel{p}{p}$ & in & 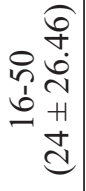 & 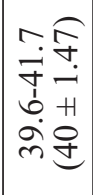 & 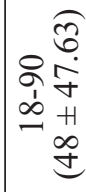 & $\begin{array}{l}\sum \\
\dot{\sigma} \\
E \\
E \\
\Xi\end{array}$ & $\infty$ & 8 & $\infty$ & 8 & 0 & $\begin{array}{l}\text { IS } \\
\infty \\
\infty \\
\end{array}$ & 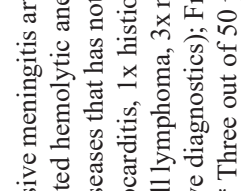 \\
\hline $\begin{array}{l}\frac{\Omega}{n} \\
\frac{2}{11} \\
\tilde{\omega}\end{array}$ & $n$ & 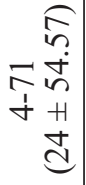 & 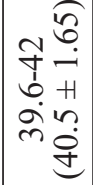 & 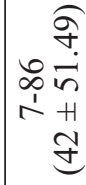 & 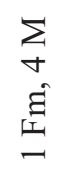 & 8 & ㅇ & શે & 8 & 0 & 坞 & 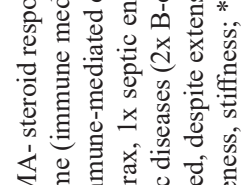 \\
\hline$\tilde{a}$ & $N$ & 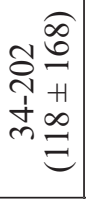 & 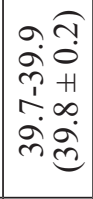 & $\begin{array}{l}+1 \\
0 \\
= \\
\infty \\
1 \\
\text { c }\end{array}$ & $\sum_{\lambda}$ & ¿ & @ & 0 & 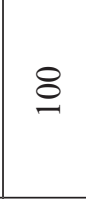 & in & $\stackrel{5}{8}$ & 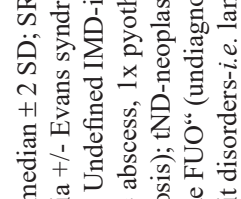 \\
\hline 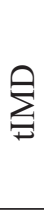 & $\stackrel{\infty}{\sim}$ & 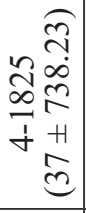 & $\begin{array}{ll}n & 0 \\
\ddots & 0 \\
\dot{y} & 0 \\
0 & +1 \\
0 & 0 \\
\dot{0} & 0 \\
\dot{m} & \dot{0} \\
\end{array}$ & 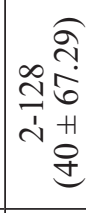 & $\begin{array}{l}\Sigma \\
= \\
E \\
\Xi\end{array}$ & ते & $\stackrel{+}{\stackrel{i}{R}}$ & $\stackrel{\sim}{\sim}$ & $\stackrel{\text { mे }}{\infty}$ & $\frac{\Delta}{\pi}$ & 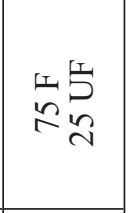 & 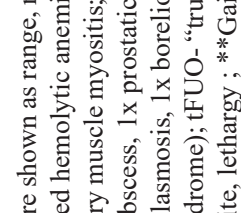 \\
\hline & 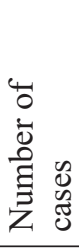 & 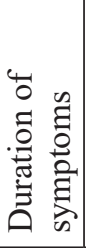 & 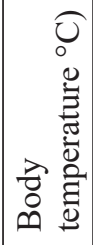 & 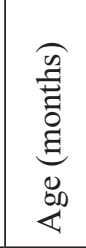 & 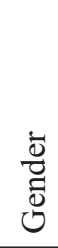 & 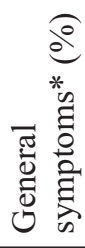 & 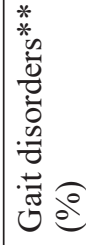 & 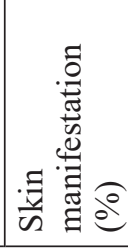 & 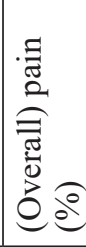 & 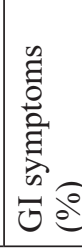 & $\begin{array}{l}\widehat{o} \\
0 \\
0 \\
0 \\
0 \\
0 \\
0\end{array}$ & 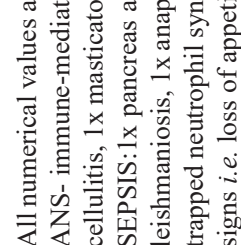 \\
\hline
\end{tabular}


Table 4. Age distribution in the investigated population

\begin{tabular}{|l|c|c|c|c|c|c|c|c|}
\hline \multirow{2}{*}{$\begin{array}{l}\text { Age group } \\
\text { (months) }\end{array}$} & \multicolumn{2}{|c|}{ Juvenile (2-18) } & \multicolumn{2}{c|}{ Young (19-48) } & \multicolumn{2}{c|}{ Middle age (49-84) } & \multicolumn{2}{c|}{ Older (85-128) } \\
\cline { 2 - 10 } & $\mathrm{n}$ & $\%$ & $\mathrm{n}$ & $\%$ & $\mathrm{n}$ & $\%$ & $\mathrm{n}$ & $\%$ \\
\hline IMD & 11 & 61.1 & 8 & 57.14 & 5 & 41.67 & 4 & 66.67 \\
\hline ID & 4 & 22 & 5 & 35.72 & 2 & 16.67 & 1 & 16.67 \\
\hline ND & 0 & 0 & 0 & 0 & 4 & 33.3 & 1 & 16.67 \\
\hline CD & 2 & 11 & 0 & 0 & 0 & 0 & 0 & 0 \\
\hline tFUO & 1 & 5.56 & 1 & 7.14 & 1 & 8.33 & 0 & 0 \\
\hline Total & 18 & 36 & 14 & 28 & 12 & 24 & 6 & 12 \\
\hline
\end{tabular}

IMD-immune-mediated diseases, ID-infectious diseases, ND-neoplastic diseases, CD-congenital diseases, tFUO -"true FUO": fever of unknown origin (dogs with no diagnosis established despite extensive diagnostics), n-number of dogs

Table 5. Effectiveness of diagnostic procedures in the investigated population

\begin{tabular}{|c|c|c|c|c|}
\hline \multicolumn{2}{|c|}{ Diagnostic procedure } & $\begin{array}{l}\text { Total number of } \\
\text { procedures performed }\end{array}$ & $\begin{array}{l}\text { Number of procedures } \\
\text { that confirmed the final } \\
\text { diagnosis }\end{array}$ & $\begin{array}{l}\text { Portion of procedures } \\
\text { that confirmed the final } \\
\text { diagnosis }(\%)\end{array}$ \\
\hline \multicolumn{2}{|c|}{ Hematology } & 222 & 3 & 1.35 \\
\hline \multicolumn{2}{|c|}{ Biochemistry } & 196 & 0 & 0 \\
\hline \multicolumn{2}{|c|}{ Urinalysis } & 20 & 0 & 0 \\
\hline \multicolumn{2}{|c|}{ Radiography } & 39 & 2 & 5.13 \\
\hline \multicolumn{2}{|c|}{ Ultrasound } & 25 & 6 & 24 \\
\hline \multicolumn{2}{|c|}{ Cytology (and/or pathohistology) } & 38 & 29 & 76.32 \\
\hline \multicolumn{2}{|c|}{ Bacteriology/culture } & 20 & 0 & 0 \\
\hline \multicolumn{2}{|c|}{ Hemoculture } & 3 & 0 & 0 \\
\hline \multirow{6}{*}{$\begin{array}{l}\text { Specific } \\
\text { tests }\end{array}$} & $4 \mathrm{DX}$ & 21 & 1 & 4.8 \\
\hline & Leptospirosis & 8 & 0 & 0 \\
\hline & Leishmaniosis & 7 & 3 & 42.86 \\
\hline & PCR - GI Viruses and VBD & 15 & 2 & 13.33 \\
\hline & ANA & 8 & 5 & 62.5 \\
\hline & RF & 4 & 0 & 0 \\
\hline \multicolumn{2}{|l|}{ Total } & 626 & $51 *$ & \\
\hline
\end{tabular}

GI-gastrointestinal, VBD-vector-borne diseases, ANA-antinuclear antibodies, RF-rheumatoid factor; *in some cases the diagnosis was established by a combination of two diagnostic tests

Tests results. Hematology and biochemistry results are shown in Figs. 1-11. Considering packed cell volume (PCV), mean values in four out of five disease groups were below the reference range. The mean value of PCV was only in the reference range in neoplastic diseases (Fig. 2). The mean values of white blood cell count (WBC), segmented neutrophils percentage and non-segmented (band) neutrophils percentage were above the reference range in all disease groups, except congenital diseases, in which these values were far below the reference range (Fig. 4). In contrast, the lymphocyte percentage was below the reference range in all groups, except congenital diseases, in which it was extremely high (Figs. 5-7). 


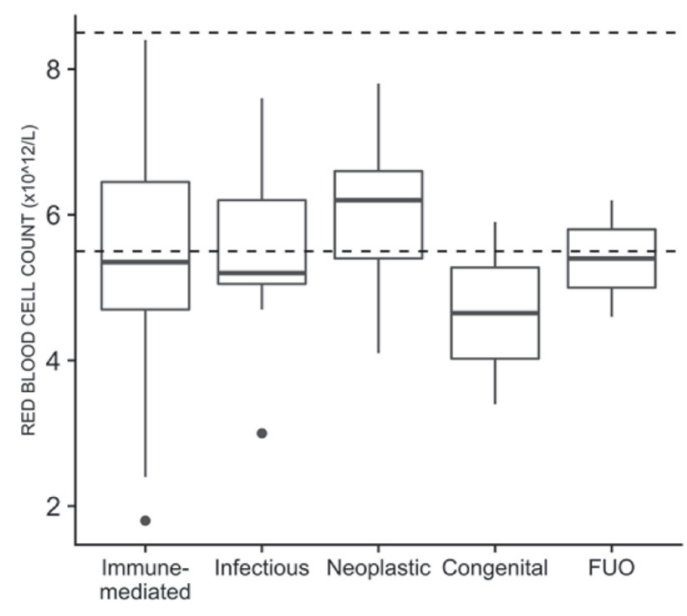

Fig. 1. Red blood cell count in the investigated population (the area between dashed lines represents the

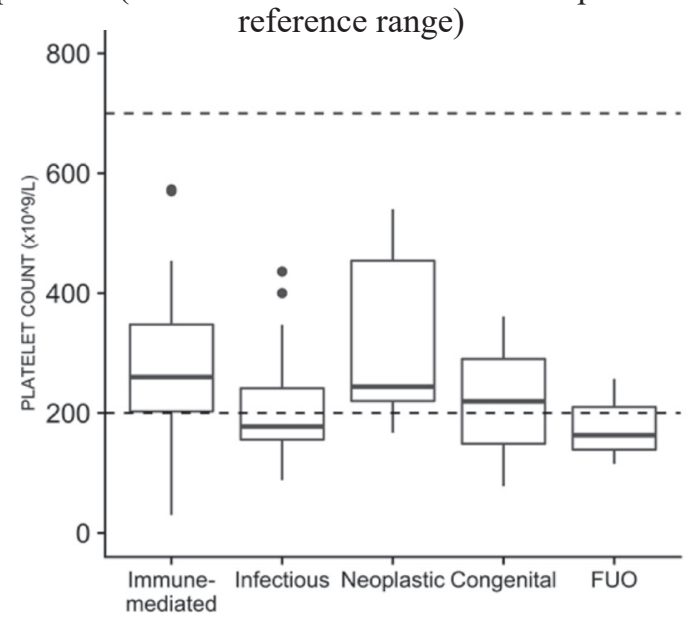

Fig. 3. Platelet count in the investigated population (the area between dashed lines represents reference the range)

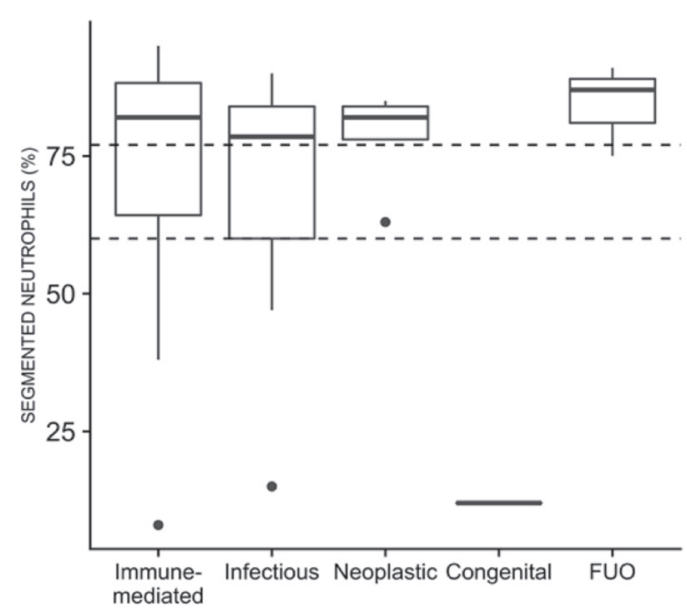

Fig. 5. Percentage of segmented neutrophils in the investigated population (area between dashed lines represents the reference range)

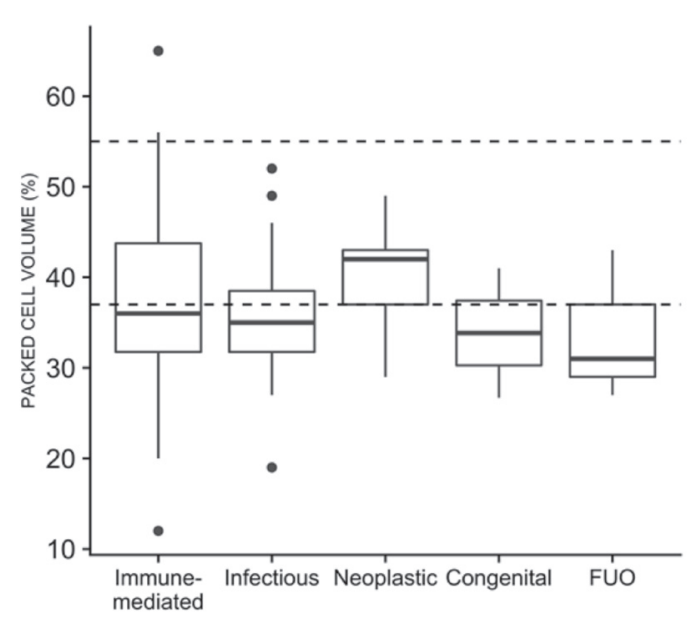

Fig. 2. Packed cell volume in the investigated population (the area between dashed lines represents the

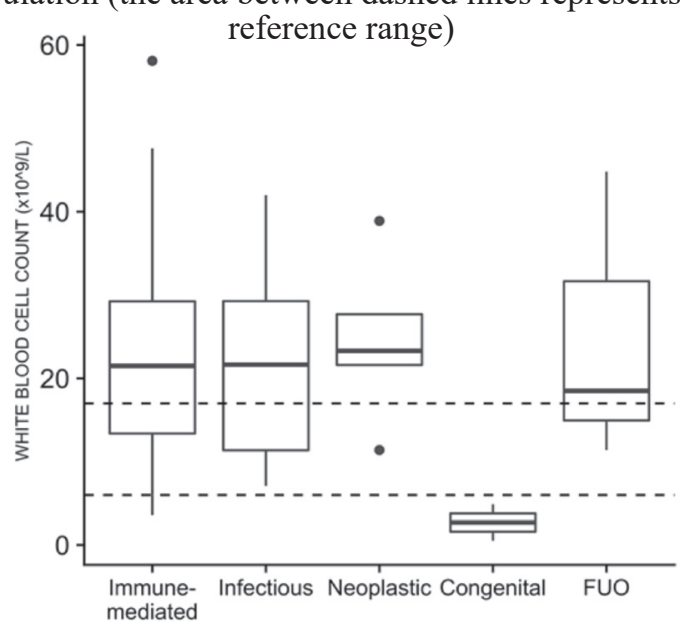

Fig. 4. White blood cell count in the investigated population (the area between dashed lines represents the

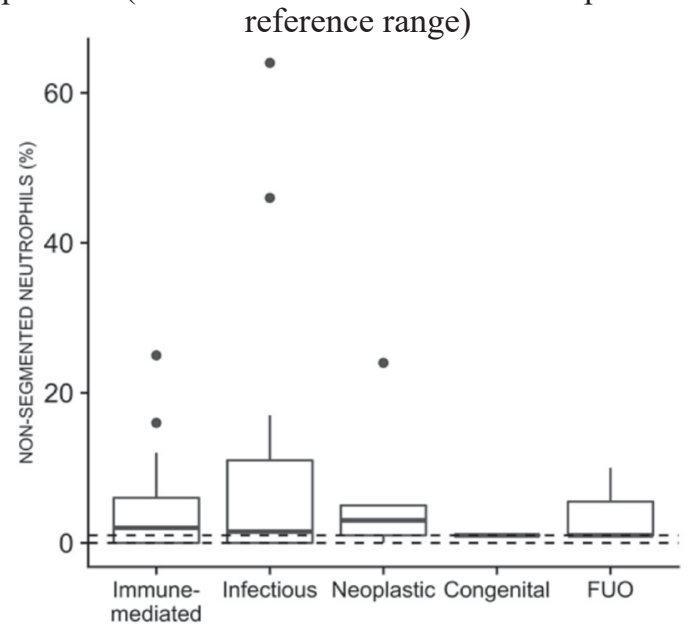

Fig. 6. Percentage of non-segmented neutrophils in the investigated population (the area between dashed lines represents the reference range) 


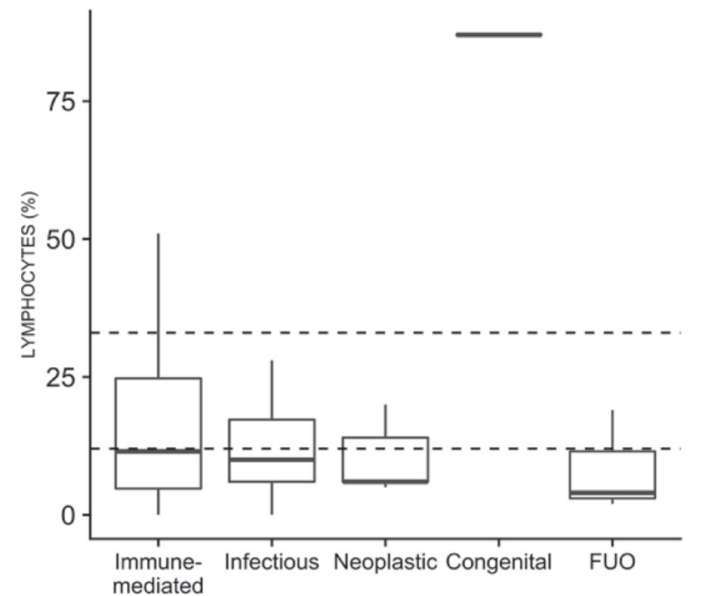

Fig. 7. Percentage of lymphocytes in the investigated population (the area between dashed lines represents the reference range)

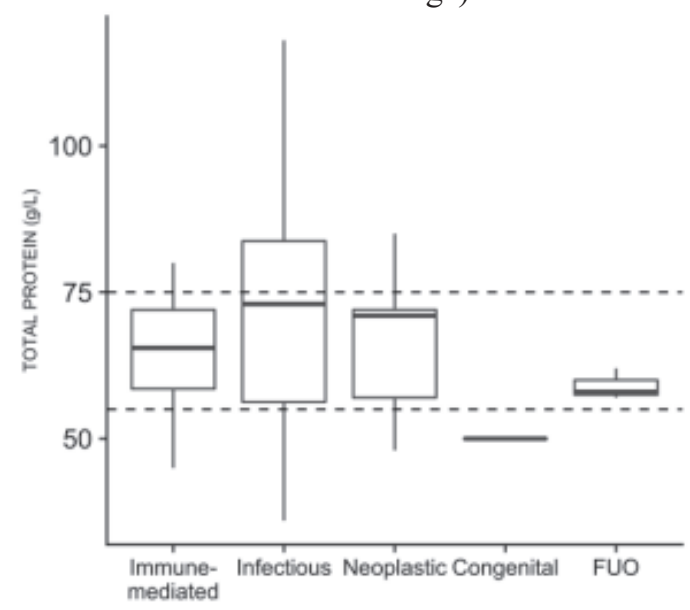

Fig. 9. Total protein concentration in the investigated population (the area between dashed lines represents the reference range)

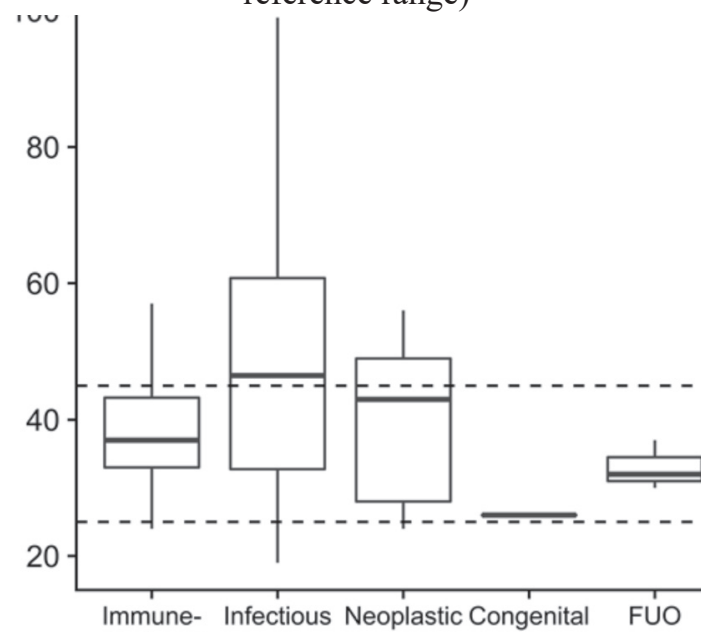

Fig. 11. Globulin concentration in the investigated population (the area between dashed lines represents the reference range)

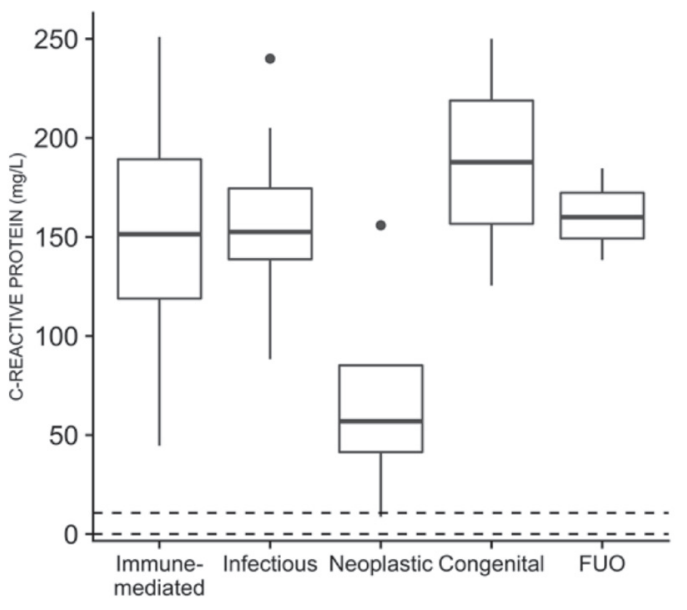

Fig. 8. C-reactive protein concentration in the investigated population (the area between dashed lines

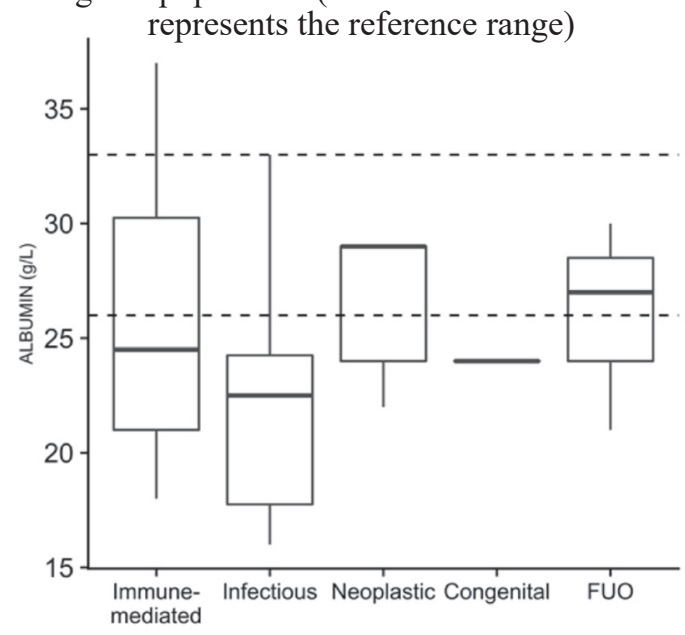

Fig. 10. Albumin concentration in the investigated population (the area between dashed lines represents the reference range)

There was no statistically significant difference between disease groups in any of the investigated hematology (Figs. 1-7) or biochemistry (Figs. 8-11) parameters. However, the concentration of C-reactive protein (CRP) was appropriately increased in all fifty cases (Fig. 8).

Duration of symptoms. The duration of clinical signs until the diagnosis/proper treatment was 4-1825 (33 \pm 590.75$)$ days, with the longest duration for undefined IMD (772-1825; mean $1298.5 \pm 1053)$ (Table 3).

Previous treatment. Only three dogs $(6 \%)$ did not receive any antibiotic treatment before admission, while the vast majority $(72 \%)$ received 
several different antibiotics. Non-steroidal antiinflammatory drugs (NSAIDs) were administered in $36 \%$ of dogs before admission. The majority of dogs received different types of supportive treatment before admission (i.e. fluid therapy, vitamins, gastric protectants etc.).

Outcome. The outcome was favorable in $62 \%$, unfavorable in $32 \%$ and unknown in $6 \%$ of all cases. The most favorable outcome was noted in IMD $(75 \%)$, and the least favorable in ND $(20 \%)$ (Table 3).

\section{Discussion}

Since dealing with FUO is extremely time (and money) consuming for both the clinician and the owner, and often life threatening for the patient, thus involving a large amount of frustration, the main goal in handling FUO is to convert "FUO" into a definitive diagnosis, while minimizing expense, the invasiveness of diagnostics and the patient's discomfort.

In human medicine, FUO is strictly defined as a fever $\geq 38.3{ }^{\circ} \mathrm{C}$ that lasts for 3 weeks or more that remains undiagnosed after 3 days of in-hospital testing, or during two or more outpatient visits (PETERSDORF and BEESON, 1961; DURACK and STREET, 1991) while in veterinary medicine FUO is still loosely defined and in a "grey zone". FUO in dogs is usually described as a fever higher than $39.7^{\circ} \mathrm{C}$, up to $40.0^{\circ} \mathrm{C}$, with no specific duration (DUNN and DUNN, 1998; BATTERSBY et al., 2006) or with various different durations (RAMSEY and TASKER, 2017). A large number of diseases can cause fever in humans (KNOCKAERT et al., 2003) as well as in dogs, so the identification of its cause requires a thorough, logical and consistent diagnostic approach to each patient.

Fifty dogs met the inclusion criteria among which three remained undiagnosed at the end of investigation ("true FUO"). Compared to other studies $(19 \%$ in the study by DUNN and DUNN, $1998 ; 22.7 \%$ in the study by BATTERSBY et al., 2006; $28 \%$ in the study by CHERVIER et al., 2012; and $17.9 \%$ in the study by BLACK et al., 2018), the lower percentage (6\%) of "true FUO" dogs in this study is most probably the result of a different diagnostic approach. Therefore, these results should be compared with caution, considering the fact that in the aforementioned studies, the undiagnosed dogs were not actually dogs with "true FUO", but rather dogs without definitive diagnosis in which the fever resolved either spontaneously or, in the vast majority of cases, after empirical treatment (antibiotics/NSAIDs/steroids). For example, in the study performed by BLACK et al. (2018) 25/115 dogs were undiagnosed, with 24/25 dogs successfully treated with corticosteroids, among which 21 dogs were treated under the provisional diagnosis of SRMA based only on clinical signs and signalment, and one dog was euthanized before the diagnosis was made. These 24 or 25 dogs should not be considered either as "true FUO" or as SRMA. Also, the authors stated that in these 24 dogs, "pyrexia resolved before investigations were completed", which is controversial regarding the empirical use of corticosteroids. In the study by BATERSBY et al. (2006) in 22.7\% (15 dogs) diagnosis could not be made, and in 7 of these, trial therapy was initiated, in 7 the fever resolved spontaneously (5 of these dogs had received treatment within 24 hours prior to referral) and in 1 dog the owner refused the diagnostics. CHERVIER et al. (2012) conducted a study in which in 14/50 dogs diagnosis was not made, but it should be noted that in 13/14 dogs the owners refused diagnostics. Finally, to conclude, the difference in the number of undiagnosed patients in this study compared to other studies lies in a fact that the owner's refusal of extensive diagnostic workup and fever resolution due to treatment trial before pursuing extensive diagnostics were exclusion criteria in our study.

On the basis of the final diagnosis, 47 (94\%) dogs in our study were classified as having IMD, ID, ND or CD. Considering the above-mentioned discrepancies, when omitting the percentage of "true FUO", the proportion of cases in all four remaining groups was similar to other studies (the most frequent cause of fever was IMD followed by fever caused by ID and neoplasms, respectively). Since most of the studies showed that IMDs have an incidence at least twice as high as ID, empirical/ trial treatment with antibiotics (previously recommended and frequently used) (COUTO, 
2014) should not be the treatment of choice in FUO patients. The most frequently diagnosed disease in this study was SRMA (18\%), which is in accordance with most other studies investigating pyrexia in dogs (BATERSBY et al., 2006; BLACK and al., 2018). The consistency in SRMA prevalence among different studies of pyrexia in dogs is not unexpected considering the fact that the odds of SRMA development are breed-related but not associated with geographical, demographic, social or environmental factors (ROSE et al., 2014). Also, as in other studies, IMPA (12\% in this study) is the second most frequent disease observed (BATERSBY et al., 2006; BLACK and al., 2018).

Regarding IDs, we categorized them into 2 groups: 1) systemic/septic infections and 2) VBD. Many bacterial and fungal infections can be hidden due to occult localization and therefore are both hard to diagnose and treat properly because their causative agents are not sensitive to the standard, commonly used empirical antibiotic therapy. Therefore, it is not surprising that these patients will have fever until extensive diagnostics are performed and both localization and microorganisms are recognized. Vector borne diseases are related to geographical and environmental factors, and their treatment also includes specific drugs not commonly used in general veterinary practice. While in human medicine IDs represent the vast majority of FUO cases in children (CHUSID, 2017), in dogs ID are much less reported as the cause of FUO than IMD. The occurrence of ND in human medicine is much more frequent than in veterinary medicine (ARMENTANO, 2016). Fever in these cases can be part of a paraneoplastic syndrome, as well as a consequence of various complications related to ND. In this study we detected $10 \%$ of ND causing FUO. Since pancreatic tumors are one of the rarest tumors in dogs, it was surprising that $3 / 5$ cases due to ND were of pancreatic origin. This indicates that pancreatic tumors could cause fever with higher frequency than other neoplasms. The low incidence of CD in dogs with FUO in this study (4\%) and other similar studies $(2.9 \%$ in the study by BLACK et al., 2018) was expected, due to the fact that CDs are rare, as well as breed and genotype dependent.
The age distribution was wide (2-128 months; mean 3.6 years) but juvenile and young dogs prevailed (64\%) (Table 4). These data are similar to other published data regarding fever in dogs (mean: 4.6 years in the study by DUNN and DUNN, 1998; 3.1 years in the study by BATTERSBY et al., 2006; and 2.25 years in the study by CHERVIER et al., 2012). The prevalence of IMD, followed by ID in almost all age groups (Tables 3 and 4) was also noted in other studies. The predomination of young dogs having FUO is not unexpected due to the fact that a large number of IMDs and almost all CDs usually occur early in life. Furthermore, it is a well-known fact that IDs are much more common in younger populations, which can be explained by immune status development. The only difference considering the distribution of diagnoses between age groups is that in our study NDs were most common in middle aged dogs, while in the study by DUNN and DUNN (1998) the incidence of ND was highest in the older group. There was no significant gender prevalence, which is in agreement with the data previously published (DUNN and DUNN, 1998; BATTERSBY et al., 2006; CHERVIER et al., 2012). Australian Shepherd, Basset, Beagle, Bernese Mountain Dog, Chow Chow, English Setter, German Shepherd, Greyhound, Puli and Tornjak were breeds that were overrepresented in the investigated population compared to the hospital population (Table 2). One should be cautious when interpreting these results since some breeds are extremely rare in the hospital population (i. e. Australian Shepherd, Basset, Chow Chow, English Setter, Greyhound, Puli and Tornjak - all below $0.2 \%$ of the hospital population) while the predominance of more popular breeds (i. e. German Shepherd, Beagle and Bernese Mountain Dog) in the FUO population represents a more reliable result. This is not surprising because all of these three breeds are prone to develop IMD (i.e. Beagles and Bernese Mountain Dog for SRMA and German Shepherd for IMPA) (ROSE et al., 2014; DUNN and DUNN, 1998).

One of the main problems in diagnosing the underlying cause of FUO is the non-specificity of the clinical presentation, which was also the case in the present study. However, the high percentage of gait disorders and/or pain (especially neck pain) 
in young dogs with fever should raise the suspicion of IMD (i.e. SRMA, IMPA) (Table 3). The high frequency of SRMA and IMPA cases in studies of pyrexia/FUO in dogs could also be attributed to the reluctance of general veterinary practitioners to perform certain diagnostic procedures, such as CSF or synovial fluid sampling, which results in the clustering of these patients within referral centers.

Since there is no accepted strict definition of FUO in veterinary medicine, FUO is usually declared in any febrile patient that has not been diagnosed after performing routine diagnostic tests, where the term "routine" is highly variable between different authors. Consequently, diagnostic workup is usually long lasting and unpredictable, making the owners dubious and frustrated. Still, an individual, thorough approach in each FUO patient (based on history, clinical examination, and different diagnostic procedures depending on most probable differentials, as well as on numerous reevaluations) is worthwhile in most cases. Nevertheless, this study also showed that an average of 12.5 tests per patient was necessary to determine a final diagnosis. Most authors agree that the aforementioned initial workup in febrile patients should include a thorough history, clinical examination, $\mathrm{CBC}$, biochemistry, urinalysis and radiography, which already represents 5 (basic) procedures, rarely sufficient in reaching a diagnosis. Anyhow, although rarely diagnostic, these tests should not be neglected because they direct the clinician towards further diagnostic procedures that should follow. This was also confirmed in this study. For example, radiography was crucial in reaching an exact diagnosis in just two cases (diagnostic success $5.1 \%$ ) of discospondylitis (Table 5) while cytology and/or PHD were crucial in 27 cases (diagnostic success 76.32\%) (Table 5). However, radiography frequently revealed the site that should be sampled for cytology/PHD (i.e. IMPA, pyothorax, lymphoma). Likewise, although there were no significant differences in hematological parameters between disease groups, specific patterns could be used as guidelines towards diagnosis. For example, a patient with marked leukopenia combined with neutrophil percentage (segmented and non-segmented) below the reference range, and lymphocyte percentage above the reference range, should be tested for a congenital disease. As in other reports (CHERVIER et al., 2012), cytology and/or PHD had the highest, while bacteriology (Table 5) the lowest percentage of success. This data are not surprising due to the fact that FNA is performed when there is an objective indication (revealed by other procedures), while bacteriology is most often done in order to exclude bacterial infection (i.e. bacteriological examination of CSF to exclude bacterial meningitis prior to corticosteroid treatment for SRMA). Also, the relatively high proportion of effectiveness of specific tests in confirming the final diagnosis is partly on account of their prudential application. Finally, since there is no diagnostic gold standard for FUO, there is no better way of managing FUO than by using the already proven multistage approach: initial diagnostics followed by a systematic, rational and objective choice of additional diagnostic procedures. This type of approach unfortunately results in the use of a large number of different tests for each patient, but has the highest odds for making a final diagnosis, and contributes to the patient's welfare.

\section{Conclusion}

In conclusion, FUO still remains a real challenge in veterinary medicine regarding the unclear definition and failure in recognizing and treating the underlying disease. This is also supported by the results of our study considering the long duration of symptoms and unsuccessful prior treatments. The long duration of clinical signs in numerous cases (the longest duration being 1825 days), during which patients were treated with numerous antibiotics, NSAIDs and other futile (inefficient) treatments, showed that veterinary practitioners still neither understand the concept of FUO (which should better be termed as fever of origin yet to be discovered) nor follow the diagnostic guidelines. Since only $24 \%$ of FUOs were due to ID (among which half were due to VBD, thus requiring specific treatment) it is obvious that treating a FUO patient with broad-spectrum antibiotics, in the best case scenario, would be efficient in only $12 \%$ of cases in this study. On the other hand, despite the fact that most recent studies have confirmed that the majority of FUO are caused by IMD, a trial treatment with 
corticosteroids, before pursuing diagnostics, would seriously compromise patient welfare (or may even cause death) in $24 \%$ of patients in this study. Giving corticosteroids without excluding a possible infection, also compromises the patient's welfare and is in direct opposition to the fundamental principle of medicine: "First, do no harm." Since trial/empirical treatment in many cases showed that it is at least useless if not harmful, as well as prolonging the time to diagnosis, it is strongly not recommended in FUO patients (ARMENTANO, 2016). To summarize, the general approach to FUO patient should always be supported by awareness that in most cases the etiology of FUO is not a rare disease but rather a common disease presenting in a nonspecific or atypical fashion.

\section{Acknowledgements}

The authors are grateful to their colleagues from the Clinic for Internal Diseases, Veterinary Faculty, University of Zagreb, for their devoted efforts in managing the patients.

\section{References}

ARMENTANO, F. (2016): Fever of unknown origin. In: Clinical Medicine of the Dog and Cat, $3^{\text {rd }}$ ed. (Michael S., F. P. Gaschen, Eds.), CRC Press, Boca Raton, pp. 61-68.

BATTERSBY, I. A., K. F. MURPHY, S. TASKER, K. PAPASOULIOTIS (2006): Retrospective study of fever in dogs: laboratory testing, diagnoses and influence of prior treatment. J. Small Anim. Pract. 47, 370-376.

DOI: $10.1111 /$ j.1748-5827.2006.00042.x

BLACK, V. L., F. J. S. WHITWORTH, S. ADAMANTOS (2018): Pyrexia in juvenile dogs: a review of 140 referred cases. J. Small Anim. Pract. 60, 116-120.

DOI: 10.1111/jsap.12938

BRKLJAČIĆ, M., V. MATIJATKO, I. KIŠ, N. KUČER, J. FORŠEK, R. BARIĆ RAFAJ, D. GRDEN, M. TORTI, I. MAYER, V. MRLJAK (2010): Molecular evidence of natural infection with Babesia canis canis in Croatia. Acta Vet. Hung. 58, 39-46.

DOI: $10.1556 /$ avet.58.2010.1.4

CHERVIER, C., L. CHABANNE, M. GODDE, M. I. RODRIGUEZ-PIÑEIRO, B. L. DEPUTTE, J. L. CADORÉ (2012): Causes, diagnostic signs, and the utility of investigations of fever in dogs: 50 cases. Can. Vet. J. 53, 525-530.

CHOW, A, J. L. ROBINSON (2011): Fever of unknown origin in children: a systematic review. World J. Pediatr. 7, 5-10.

CHUSID, M. J. (2017): Fever of unknown origin in childhood. Pediatr. Clin. North Am. 64, 205-230.
COUTO, C. G. (2014): Fever of undetermined origin. In: Small Animal Internal Medicine, $5^{\text {th }}$ ed. (Nelson, R. W., C. G. Couto, Eds.), Elsevier, St. Louis, pp. 1279-1282.

DEŽĐEK, D., L. VOJTA, S. ĆURKOVIĆ, Z. LIPEJ, Ž. MIHALJEVIĆ, Ž. CVETNIĆ, R. BECK (2010): Molecular detection of Theileria annae and Hepatozoon canis in foxes (Vulpes vulpes) in Croatia. Vet Parasitol. 20, 172, 333-6.

DOI: 10.1016/j.vetpar.2010.05.022

DUNN, K. J., J. K. DUNN (1998): Diagnostic investigations in 101 dogs with pyrexia of unknown origin. J. Small Anim. Pract. 39, 574-580.

DOI: $10.1111 / \mathrm{j} .1748-5827.1998 . t b 03711 . x$

DURACK, D. T., STREET, A. C. (1991): Fever of unknown origin: Reexamined and redefined. Curr. Clin. Top. Infect. Dis. $11,35-51$.

JURKOVIĆ, D., A. BECK, D. HUBER, Ž. MIHALJEVIĆ, A. POLKINGHORNE, F. MARTINKOVIĆ, D. LUKAČEVIĆ, M. PILAT, R. BREZAK, S. BOSNIĆ, R. BECK (2019): Seroprevalence of vector-borne pathogens in dogs from Croatia. Parasitol. Res. 118, 347-352.

DOI: 10.1007/s00436-018-6129-7

KNOCKAERT, D. C., S. VANDERSCHUEREN, D. BLOCKMANS (2003): Fever of unknown origin in adults: 40 years. J. Intern. Med. 253, 263-75.

DOI: 10.1046/j.1365-2796.2003.01120.x

MRLJAK, V., J. KULEŠ, Ž. MIHALJEVIĆ, M. TORTI, J. GOTIĆ, M. CRNOGAJ, T: ŽIVIČNJAK, I. MAYER, I. ŠMIT, M. BHIDE, R. BARIĆ RAFAJ (2017): Prevalence and geographic distribution of vector-borne pathogens in apparently healthy dogs in Croatia. Vector Borne Zoonotic Dis. $17,398-408$.

DOI: $10.1089 /$ vbz.2016.1990

PETERSDORF, R. G., P. B. BEESON (1961): Fever of unexplained origin: Report on 100 cases. Medicine 40, 1-30.

DOI: 10.1097/00005792-196102000-00001

RAMSEY, I. K., S. TASKER (2017): Fever. In: Textbook of Veterinary Internal Medicine: Diseases of the Dog and the Cat, $8^{\text {th }}$ ed. (Ettinger, S. J., E. C. Feldman, E. Côté, Eds.), Elsevier, St. Louis, pp. 679-694.

ROSE, J. H., M. KWIATKOWSKA, E. R. HENDERSON, N. GRANGER, J. K. MURRAY, T. R. HARCOURTBROWN (2014): The impact of demographic, social, and environmental factors on the development of steroidresponsive meningitis-arteritis (SRMA) in the United Kingdom. J. Vet. Intern. Med. 28, 1199-202.

DOI: $10.1111 /$ jvim. 12360

ŽIVIČNJAK, T., F. MARTINKOVIĆ, A. MARINCULIĆ, V. MRLJAK, N. KUČER, V.MATIJATKO,Ž. MIHALJEVIĆ, R. BARIĆ-RAFAJ (2005): A seroepidemiologic survey of canine visceral leishmaniosis among apparently healthy dogs in Croatia. Vet. Parasitol. 15, 35-43.

DOI: 10.1016/j.vetpar.2005.04.036 
Received: 1 October 2019

Accepted: 12 December 2019

BRKLJAČIĆ, M., M. CRNOGAJ, I. KIŠ, K. BOJANIĆ, F. KAJIN, I. ŠMIT, M. TORTI, I. HARAPIN, V. МАТІЈАТКО: Vrućica nepoznata uzroka u pasa: 50 slučajeva. Vet. arhiv 90, 255-269, 2020. SAŽETAK

Premda termin vrućica nepoznatog uzroka (VNU) u veterinarskoj medicini još uvijek nema točno određenu definiciju ni kriterije klasifikacije, te se vrlo često liberalno upotrebljava u febrilnih pacijenata u kojih nije postavljena egzaktna dijagnoza nakon rutinske dijagnostičke obrade, VNU bi trebalo rezervirati za one pacijente u kojih ni nakon opsežne dijagnostike nije otkriven uzrok, tj. postavljena konačna dijagnoza. Ciljevi ovog istraživanja bili su istražiti pojavnost VNU-a kao i različitih dijagnoza u pasa s vrućicom, kliničke simptome i njihovo trajanje do postavljanja dijagnoze i početka ciljanog liječenja, te ishode liječenja, odnosno bolesti. Također, svrha ovog istraživanja bila je istražiti prosječan broj dijagnostičkih postupaka (i njihovu učinkovitost) potrebnih za postavljanje konačne dijagnoze, s ciljem kreiranja dijagnostičkog plana u pasa s VNU-om u Hrvatskoj. U 94 \% pasa (47/50) postavljena je konačna dijagnoza pomoću ukupno 626 dijagnostičkih postupaka. Najčešće su bile zastupljene imunosno posredovane bolesti (56\%), zatim infekcijske (24\%), neoplastične (10\%) i kongenitalne (4\%), dok je pravi VNU bio zastupljen u samo $6 \%$ slučajeva. Tjelesna temperatura kretala se od 39,6 do $42,0{ }^{\circ} \mathrm{C}$. Najčešći su klinički znakovi bili nespecifični (gubitak apetita i letargija u $88 \%$, bolnost u $78 \%$ te poremećaji kretanja u $60 \%$ slučajeva). Najčešći je dijagnostički postupak bila hematološka pretraga, a najrjeđi hemokultura. Citološka i/ili patohistološka pretraga pokazale su se najučinkovitijima u postavljanju konačne dijagnoze (76,32 \%). Trajanje simptoma do postavljanja dijagnoze, odnosno početka adekvatnog liječenja iznosilo je od 4 do 1825 dana. Ishod je u $62 \%$ pasa bio povoljan, u $32 \%$ nepovoljan, dok je u $6 \%$ ostao nepoznat.

Ključne riječi: vrućica nepoznata uzroka; vrućica; pireksija; pas 
